\title{
Global Angular Momentum Fluxes in Height Coordinates
}

\author{
JOSEPH EGGER \\ Meteorologisches Institut, Universität München, Munich, Germany \\ KLAUS-PETER HOINKA \\ Institut für Physik der Atmosphäre, DLR, Oberpfaffenhofen, Germany
}

(Manuscript received 26 May 2010, in final form 1 December 2010)

\begin{abstract}
While time and zonal mean budgets of axial angular momentum (AAM) have been presented in pressure coordinates and also in isentropic coordinates, AAM budgets in height coordinates have not been published yet. The results of such an analysis on the basis of the 40-yr European Centre for Medium-Range Weather Forecasts (ECMWF) Re-Analysis (ERA-40) winter data are presented in this paper, which includes explicitly evaluated vertical eddy fluxes of momentum and mass as new features. As expected, AAM fluxes related to the Hadley cell are dominant. Transient vertical AAM fluxes are directed upward at the midlatitudes. Transient mass transports are not negligible, while triple terms are unimportant. Problems with the global balance of torques acting at the surface are discussed as well as those of mass conservation.
\end{abstract}

\section{Introduction}

Axial angular momentum (AAM) is a key variable in large-scale dynamics so that AAM budgets are indispensable for understanding the global circulation. Correspondingly, various AAM budgets have been evaluated from data. Of course, the results depend on the coordinate system chosen for the analysis. The investigations of Starr and White (1951), Hantel and Hacker (1978), and Oort and Peixoto (1983; see also Peixoto and Oort 1992) were performed in pressure $p$ coordinates while Gallimore and Johnson (1981) and Johnson (1980a) based their calculations on isentropic $\theta$ coordinates. The tropical Hadley circulation is the dominant feature in $p$ coordinates. Transient meridional eddy fluxes are directed poleward. These findings led to the generally accepted qualitative picture that the AAM removed in the midlatitudes by surface friction is replenished by eddy fluxes out of the tropics (e.g., Holton 1992, his Fig. 10.10). Thus, vertical fluxes in midlatitudes would have to be directed downward for this concept to be convincing. The coarsely resolved results of Hantel and Hacker (1978) indicate

Corresponding author address: Joseph Egger, Meteorological Institute, University of Munich, Theresienstr. 37, Munich 80333, Germany.

E-mail: j.egger@lrz.uni-muenchen.de that the balance is achieved by the mean motion. The vertical eddy fluxes have been derived so far only by residual methods. It is, therefore, mandatory to evaluate vertical eddy fluxes directly and relate them to the mean circulation fluxes (see also Trenberth 1987 for critical comments on the residual methods). Modern reanalysis datasets allow us to evaluate all AAM fluxes directly. The ultimate answer with respect to the balance of the various torques via AAM fluxes has to come from the calculation of an AAM streamfunction. An approximate solution has been presented by Oort and Peixoto (1983) who replaced the earth's surface by the $1000-\mathrm{hPa}$ surface.

A rather different picture emerges in $\theta$ coordinates where one direct circulation cell with two centers spans the Northern Hemisphere in winter [see also Held and Schneider (1999)]. AAM is brought down from the upper branch mainly by pressure torques (i.e., by eddy motion). The available evidence appears to suggest that midlatitude torques at the surface are balanced in the $\theta$ system by southward fluxes and downward internal torques, but a streamfunction pattern as in Oort and Peixoto (1983) has not been presented as yet in $\theta$ coordinates. As a matter of fact, it is difficult to connect equatorial surface torques with those in midlatitudes in a coordinate system where the intersections of coordinate surfaces with the ground move rapidly and widely. 
To the best of our knowledge, an AAM budget in height $z$ coordinates has as yet not been published. The role of density variations in AAM budgets can be investigated conveniently in $z$ coordinates. This is a main motivation for conducting this analysis that allows us to relate AAM transports in a more comprehensive way to synoptic eddy activity. The contribution of density to AAM almost disappears in $p$ coordinates because the mass between $p$ surfaces remains unaltered in a hydrostatic atmosphere. Variations of the total mass of columns are reflected in those of the surface pressure. It is, however, customary to neglect such effects. It is a further advantage of the $z$ system that the coordinate surfaces are fixed in space and time. The treatment of the lower boundary poses no problem in $z$ coordinates except for mountains. Connection of surface torques via the streamfunction does not pose a conceptual problem.

The angular momentum equation in the spherical $(\lambda, \varphi$, z) system is

$$
\begin{aligned}
& \frac{\partial}{\partial t}(m \rho)+\frac{1}{a \cos \varphi} \frac{\partial}{\partial \lambda}(m \rho u)+\frac{1}{a \cos \varphi} \frac{\partial}{\partial \varphi}(m \rho v \cos \varphi) \\
& +\frac{\partial}{\partial z}(m \rho w)=-\frac{\partial p}{\partial \lambda}-\frac{\partial \tau}{\partial z}
\end{aligned}
$$

where $\lambda$ is longitude, $\varphi$ is latitude,

$$
m=(u+\Omega a \cos \varphi) a \cos \varphi
$$

is the specific angular momentum with the earth's radius $a$, and $\Omega=2 \pi$ per day. The notation in (1.1) is conventional with density $\rho$ and velocity $(u, v, w)$. Moisture effects are excluded in (1.1) although the related torques are not negligible (Egger 2006). The turbulent vertical AAM flux $\tau$ by subgrid motions is assumed to contain also contributions by breaking gravity waves in the free atmosphere and is certainly important in the boundary layer. This rather uncertain term will not be included in what follows except when taking the surface friction torque into account.

It is customary and convenient to perform budgets for "annuli" extending zonally around the globe, meridionally from latitude $\varphi_{1}$ to $\varphi_{2}$, and vertically from the topography $h$ to a surface at height $z=z_{2}$. AAM budget equations are obtained by integrating (1.1) over the volume of this annulus. The result is

$$
\begin{aligned}
& \frac{d M}{d t}+F_{m}\left(\varphi_{2}, \eta, h\right)-F_{m}\left(\varphi_{1}, \eta, h\right) \\
& \quad+F_{v}\left(z_{2}, \varphi_{1}, \varphi_{2}\right)=T_{o}+T_{f}
\end{aligned}
$$

after simple manipulations involving the lower boundary condition

$$
w=\frac{d h}{d t}
$$

at $z=h$. The AAM of the ring is

$$
\begin{array}{r}
M=\int_{0}^{2 \pi} \int_{\varphi_{1}}^{\varphi_{2}} \int_{h}^{z_{2}}(m \rho) a^{2} \cos \varphi d z d \varphi d \lambda, \\
F_{m}\left(\varphi_{i}, h, z_{2}\right)=\left.\int_{0}^{2 \pi} \int_{h}^{z_{2}}(v m \rho \cos \varphi)\right|^{\varphi_{i}} a d z d \lambda
\end{array}
$$

is the meridional flux of AAM through the ring's sidewall at $\varphi=\varphi_{i}(i=1,2)$ and

$F_{v}\left(z_{2}, \varphi_{1}, \varphi_{2}\right)=\left.\int_{0}^{2 \pi} \int_{\varphi_{2}}^{\varphi_{1}}(m \rho w)\right|^{z_{2}} a^{2} \cos \varphi d \varphi d \lambda$

is the vertical flux through the upper boundary (see also Johnson 1980b). The terms on the right-hand side of (1.3) are the mountain torque $T_{o}$ and the friction torque $T_{f}$.

A well-known complication arises if time mean budgets are considered. A split of a variable

$$
b=\bar{b}+b^{\prime}
$$

in a time mean value and a deviation from there turns, for example, the vertical flux $F_{v}$ in (1.7) into

$$
\overline{F_{v}}\left(z_{2}, \varphi_{1}, \varphi_{2}\right)=\int_{0}^{2 \pi} \int_{\varphi_{1}}^{\varphi_{2}}\left[\left(\bar{w} \bar{\rho}+\overline{w^{\prime} \rho^{\prime}}\right) \bar{m}+\left(\bar{w} \overline{m^{\prime} \rho^{\prime}}+\bar{\rho} \overline{m^{\prime} w^{\prime}}\right)+\overline{\rho^{\prime} w^{\prime} m^{\prime}}\right] a^{2} \cos \varphi d \varphi d \lambda
$$

The first two terms in (1.9) describe the transport of mean specific AAM by the vertical mass flux. In particular, the transient vertical mass flux $\overline{w^{\prime} \rho^{\prime}}$ represents the conversion of potential to kinetic energy and may, therefore, be important. The next two terms are transient eddy fluxes involving zonal density fluxes $\overline{u^{\prime} \rho^{\prime}}$ and vertical AAM fluxes. It is the covariance $\overline{u^{\prime} w^{\prime}}$ that is of particular interest here. Finally, there is a triple term. Of course, the meridional mean flux $\bar{F}_{m}\left(\varphi_{1}, z_{2}, h\right)$ also contains five terms. A simplification of (1.9) could have been achieved by introducing a density weighted mean. This would lead, for example, to a lumping together of the first and third term and a disappearance of the triple term. However, important information would be lost this way. 
Let us now concentrate on time mean budgets where we may either choose the surface $z=h$ as in (1.3) as the lower boundary of a ring or a surface $z=z_{1}$ with constant height $z_{1}$. Thus,

$$
\begin{aligned}
& \overline{F_{m}}\left(\varphi_{2}, z_{1}, z_{2}\right)-\overline{F_{m}}\left(\varphi_{1}, z_{1}, z_{2}\right)+\overline{F_{v}}\left(z_{2}, \varphi_{1}, \varphi_{2}\right) \\
& \quad-\overline{F_{v}}\left(z_{1}, \varphi_{1}, \varphi_{2}\right)=0
\end{aligned}
$$

is the mean budget for a ring with lower boundary $z_{1}$ while

$$
\begin{aligned}
& \overline{F_{m}}\left(\varphi_{2}, h, z_{2}\right)-\overline{F_{m}}\left(\varphi_{1}, h, z_{2}\right)+\overline{F_{v}}\left(z_{2}, \varphi_{1}, \varphi_{2}\right) \\
& \quad=\overline{T_{o}}+\overline{T_{f}}
\end{aligned}
$$

is the budget for a ring with the earth's surface as lower boundary. Of course, the torques on the right-hand side must be evaluated for the belt $\varphi_{1}<\varphi<\varphi_{2}$. The detailed structure of the fluxes according to observations will be given in the next section. Note that we discarded the "storage terms" in (1.10) and (1.11) resulting from a time integration of the tendency of $M$ in (1.3). We will, however, briefly comment on these terms below.

\section{Results}

All data evaluations are based on the 40-yr European Centre for Medium-Range Weather Forecasts (ECMWF) Re-Analysis (ERA-40) set for the years 1958-2001. We wish to show the fluxes in (1.10) with a fairly high spatial resolution. The rings chosen have a height $D z=z_{2}-z_{1}=$ $250 \mathrm{~m}$ and a "width" $D \varphi=\varphi_{2}-\varphi_{1}=2.25^{\circ}(\sim 250 \mathrm{~km})$. The analysis is restricted to heights $z_{i} \leq 12 \mathrm{~km}$. The flux evaluations are carried out for Northern Hemisphere winter [December-February (DJF)]. The following mean vertical flux terms will be presented:

$$
\begin{aligned}
& \bar{F}_{v 1}=I_{v}(\bar{w} \bar{\rho} \bar{m}), \\
& \bar{F}_{v 2}=I_{v}\left(\overline{w^{\prime} \rho^{\prime}} \bar{m}\right), \\
& \bar{F}_{v 3}=I_{v}\left(\overline{\bar{w}} \overline{u^{\prime} \rho^{\prime}} a \cos \varphi\right), \\
& \bar{F}_{v 4}=I_{v}\left(\bar{\rho} \overline{u^{\prime} w^{\prime}} a \cos \varphi\right), \\
& \bar{F}_{v 5}=I_{v}\left(\overline{w^{\prime} \rho^{\prime} u^{\prime}} a \cos \varphi\right),
\end{aligned}
$$

where

$$
I_{v}=\int_{0}^{2 \pi} \int_{\varphi_{1}}^{\varphi_{2}}(\cdot) a^{2} \cos \varphi d \varphi d \lambda
$$

The corresponding meridional fluxes $\overline{F_{m i}}$ are obtained by replacing $w$ in (2.1) by $v$ and performing the integral

$$
I_{m}=\int_{0}^{2 \pi} \int_{z_{1}}^{z_{2}}(\cdot) a \cos \varphi d z d \lambda
$$

instead of (2.2). In the following the flux symbols $F$ and $T$ are written without bars.

A key problem with AAM balances is accuracy. For example, the ratio

$$
F_{m 4} / F_{m 1} \sim \overline{u^{\prime} v^{\prime}} /(\bar{v} \Omega a \cos \varphi)
$$

has for $\overline{u^{\prime} v^{\prime}} \sim 10 \mathrm{~m}^{2} \mathrm{~s}^{-2}, \bar{v}=1 \mathrm{~m} \mathrm{~s}^{-1}$ a value of $\sim 1 / 25$ at the midlatitudes. A balance of such fluxes requires an accuracy of $\sim 10^{-2} \mathrm{~m} \mathrm{~s}^{-1}$ for the mean velocity $\bar{v}$. That is more than we can expect even from the most recent reanalysis schemes. One may argue that it is better to replace (1.3) by the budget of relative angular momentum (e.g., Hantel and Hacker 1978), but even in that form it is difficult to achieve the needed accuracy. Moreover, the Coriolis term enters as a source in this case that destroys the elegance of (1.10). We have to be aware in addition, that all fluxes $F_{m i}$ and $F_{v i}$ are obtained by interpolation from the hybrid ERA coordinate system. That introduces further errors, in particular in mountainous terrain where constant height surfaces intersect the mountains. In other words, we cannot expect that (1.10) is satisfied by the data with great accuracy.

Figure 1a shows $F_{m 1}$, that is, the meridional mean flux of mean AAM. Of course, the meridional flux due to the Hadley cell is dominant. Fluxes peak near -100 Hadley close to the equator, the fluxes farther north are small $\left(1\right.$ Hadley $\left.=10^{18} \mathrm{~J}\right)$. The fluxes climb up to 30 Hadley in the lower troposphere near $30^{\circ} \mathrm{S}$. Note that a value $F_{m 1} \sim 50$ Hadley corresponds with $|\bar{v}| \sim 2 \mathrm{~m} \mathrm{~s}^{-1}$ near the ground at the equator.

A rather unexpected pattern is displayed in Fig. $1 \mathrm{~b}$ where the eddy mass transport $F_{m 2} \sim\left(\overline{v^{\prime} \rho^{\prime}}\right) \bar{m}$ is presented. In both hemispheres, there is an equatorward eddy mass flux that extends through the depth of the troposphere and is centered near $40^{\circ} \mathrm{N}$ and $\sim 40^{\circ} \mathrm{S}$. Amplitudes of $\sim 0.6 \mathrm{Had}-$ ley near the ground are not negligible in the midlatitudes.

The term $F_{m 3}$ (Fig. 1c) is mainly positive at the midlatitudes, but too small to be of interest. Maximum values of $\sim 0.002$ Hadley are far below the limits of accuracy of an AAM balance. The term $F_{m 4}$ (Fig. 1d) describes the well-known poleward eddy transport $\sim \overline{u^{\prime} v^{\prime}}$ of relative AAM peaking near $30^{\circ} \mathrm{N}\left(30^{\circ} \mathrm{S}\right)$ in the upper troposphere. The triple term $F_{m 5}$ is small and noisy (Fig. 1e). It does not need to be taken into consideration.

The vertical flux term $F_{v 1}$ (Fig. 2a) also mainly reflects the Hadley circulation, but the columns of vertical motion north of $30^{\circ} \mathrm{N}$ also fit, of course, the meridional fluxes in Fig. 1a. In particular, the mean flux is directed upward in 


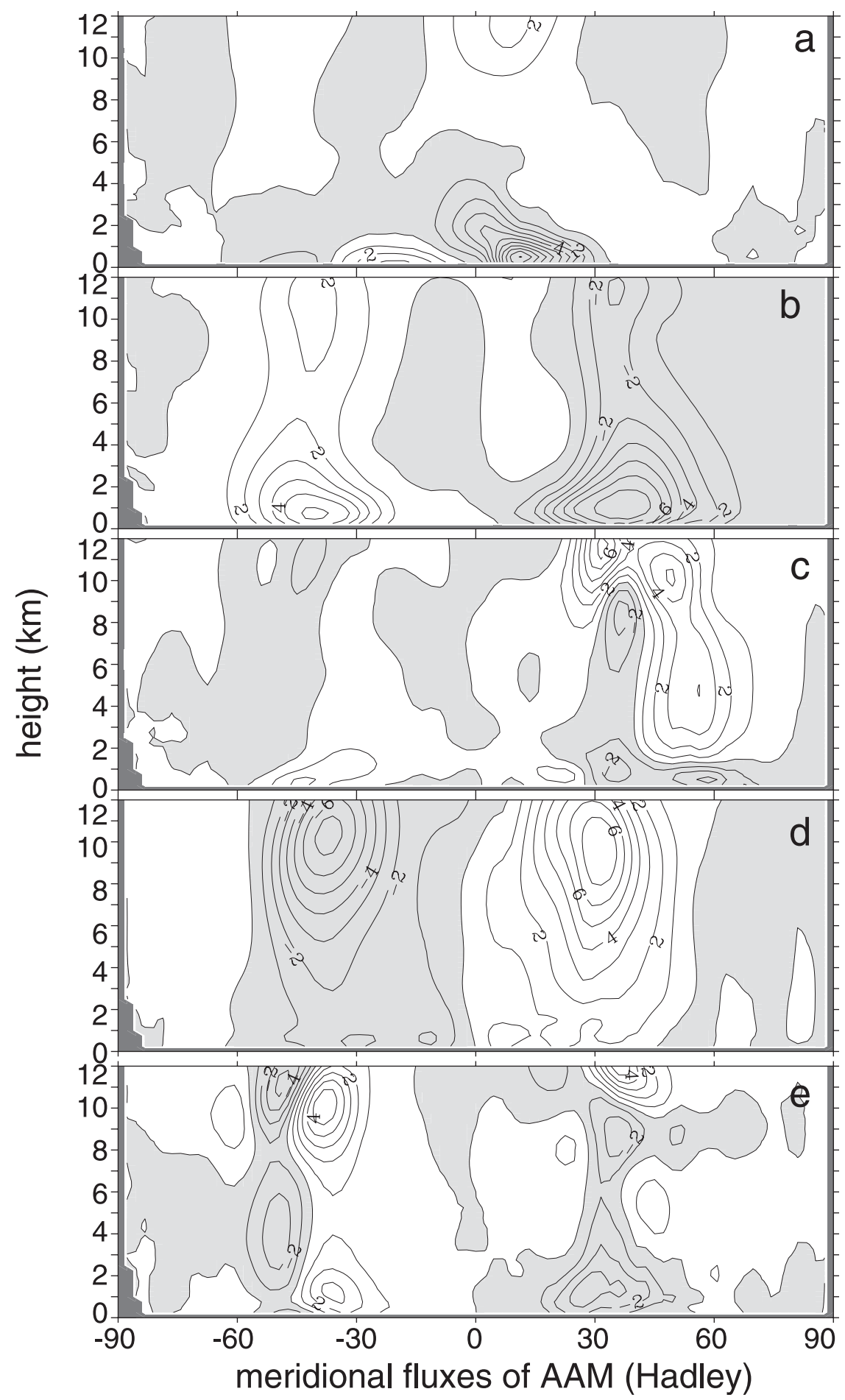

FIG. 1. Meridional fluxes of AAM with respect to zonal rings of 250-m depth in winter: (a) $F_{m 1} \sim \bar{v} \bar{\rho} \bar{m}$ in 10.0 Hadley, (b) $F_{m 2} \sim \overline{v^{\prime} \rho^{\prime}} \bar{m}$ in 0.1 Hadley, (c) $F_{m 3} \sim \bar{v} \overline{u^{\prime} \rho^{\prime}} a \cos \varphi$ in 0.0005 Hadley, (d) $F_{m 4} \sim \overline{u^{\prime} v^{\prime}} \bar{\rho} a \cos \varphi$ in 0.1 Hadley, and (e) $F_{m 5} \sim \overline{u^{\prime} v^{\prime} \rho^{\prime}} a \cos \varphi$ in 0.0005 Hadley. (1 Hadley $=10^{18} \mathrm{~J}$.) 
the belts $45^{\circ} \leq \varphi \leq 65^{\circ} \mathrm{N}$ and $70^{\circ} \leq \varphi \leq 45^{\circ} \mathrm{S}$. The eddy mass flux $\sim\left(\overline{\rho^{\prime} w^{\prime}}\right)$ (see Fig. $2 \mathrm{~b}$ ) is quite small in the tropics, but negative and not negligible in midlatitudes. As stated above, one would have expected to find negative values of $\left(\overline{\rho^{\prime} w^{\prime}}\right)$. Indeed, the maximum energy conversions occur where baroclinic activity is largest. Figures $1 \mathrm{~b}$ and $2 \mathrm{~b}$ illustrate the processes of slantwise convection in baroclinic disturbances where dense air with relatively low values of AAM is moving down toward the south while less dense air with larger angular momentum is ascending northward (e.g., Holton 1992). Of course, $\left(\overline{\rho^{\prime} w^{\prime}}\right)=0$ at $z=0$, but the display in Fig. 2b begins at the lowest level $z=250 \mathrm{~m}$. Again, there appears to be a mass balance problem because of the mean downward eddy mass transport.

It is reasonable to expect that the eddy mass fluxes $\left(\overline{\rho^{\prime} v^{\prime}}, \overline{\rho^{\prime} w^{\prime}}\right)$ are mainly due to adiabatic processes and are adapted to the mean isentropes. We find a slope angle,

$\alpha=\overline{\rho^{\prime} w^{\prime}} / \overline{\rho^{\prime} v^{\prime}}=F_{v 2} D z /\left(F_{m 2} a D \varphi\right) \sim 2-5 \times 10^{-3}$,

in the $\mathrm{NH}$ midlatitudes that is of the right order of magnitude.

The term $F_{m 3}$ (Fig. 2c) is small and negligible in analogy to $F_{v 3}$. The contribution by the vertical relative AAM flux $\sim\left(\overline{u^{\prime} w^{\prime}}\right)$ is displayed in Fig. 2d. This flux is directed upward in both hemispheres with a narrow region of negative values near $60^{\circ} \mathrm{N}$. Baroclinic eddies appear to transport relative momentum upward and are, therefore, antidiffusive. This result corroborates the coarse-grained residual results of Hantel and Hacker (1978) who diagnosed upward eddy transports in midlatitude winter. The slope of the relative AAM transports is close to (2.5). Egger and Hoinka (2010) presented a map of $\left(\overline{u^{\prime} w^{\prime}}\right)$ at a midtropospheric level and showed that the storm tracks are main regions of upward transports. It is easy to show that unstable Eady waves transport momentum downward. Thus, the observed upward momentum transport cannot be explained on the basis of simple baroclinic wave dynamics. The triple term $F_{v 5}$ is noisy and negligible (Fig. 2e). Note that $F_{v 2}$ is the only negative flux close to the ground in the belt $60^{\circ}<\varphi \leq 45^{\circ} \mathrm{S}$ and near $45^{\circ} \mathrm{N}$.

Stationarity is assumed in (1.10) and (1.11) although there is a certain mean change of the wind and mass distribution during a season that has been evaluated. As expected, the related AAM changes are small and amount to a maximum seasonal tendency of 0.02 Hadley in the midtroposphere near $20^{\circ} \mathrm{N}$ and a similar loss near the equator.

The results in Figs. 1 and 2 suggest that there are problems with mass conservation. Corresponding tests have been performed by comparing the total mass flux through a surface $\varphi=\varphi_{i}$, constant to that through the top surface in the domain north of $\varphi_{i}\left(z=12 \mathrm{~km} ; \varphi>\varphi_{i}\right)$. Maximum imbalances are found in the domain of the
Hadley cell $\left(0^{\circ} \leq \varphi_{i} \leq 30^{\circ} \mathrm{N}\right)$ where the mean vertical inflow is larger by $\sim(1-5) \times 10^{10} \mathrm{~kg} \mathrm{~s}^{-1}$. Such a maximum error corresponds roughly with a maximum systematic error of $0.1-0.2 \mathrm{~m} \mathrm{~s}^{-1}$ for the vertical mean meridional velocity (see also Trenberth 1987). A decrease of $D z$ from $D z=250 \mathrm{~m}$ to $D z=100 \mathrm{~m}$ leads to an error reduction. On the other hand, the equatorward eddy mass fluxes at the midlatitudes seen in Fig. 1b appear not to be balanced by corresponding mean flow transports. Vertical resolution has no effect on this phenomenon.

A control of (1.10) is displayed in Fig. 3. The flux differences are evaluated for each grid point at the available resolution. The resulting field is fairly noisy as one would expect and shows maximum error convergences of $\sim 1.5$ Hadley in the tropical belt close to the ground. This is quite an acceptable result given the large fluxes of $\sim 100$ Hadley there. It is not only the switch to the $z$ system that contributes here but there are also discretization errors. Note, however, that the lowest layer has been excluded in Fig. 3. An error analysis does not make much sense if a main term, namely, the stress $\tau$, is missing. Nevertheless, errors tend to be relatively large near the lower boundary. They may also reflect the impact of the mountains.

\section{Streamfunction}

The zonal mean budget is nondivergent so that the presentation of a streamfunction $\psi$ will help to clarify the distribution of total AAM fluxes (see Oort and Peixoto 1983) and the connection of sources and sinks at the surface by streamlines. With

$$
\begin{gathered}
\frac{\partial \psi}{\partial z}=\int_{0}^{2 \pi} \overline{v m \rho} a \cos \varphi d \lambda=-V, \\
a^{-1} \frac{\partial \psi}{\partial \varphi}=\int_{0}^{2 \pi} \overline{w m \rho} a \cos \varphi d \lambda=W
\end{gathered}
$$

we introduce a streamfunction that solves the "vorticity" relation:

$$
a^{-2} \frac{\partial^{2} \psi}{\partial \varphi^{2}}+\frac{\partial^{2} \psi}{\partial z^{2}}=a^{-1} \frac{\partial W}{\partial \varphi}-\frac{\partial V}{\partial z} .
$$

The right-hand side represents the zonal component of a "flux vorticity." The boundary conditions are

$$
W=T_{o}+T_{f}
$$

at $z=0, V=0$ at the Poles, and the observed profile of $W$ at $z=12 \mathrm{~km}$. Because the sum of mountain and friction torque is positive in the tropics, negative in midlatitudes, 


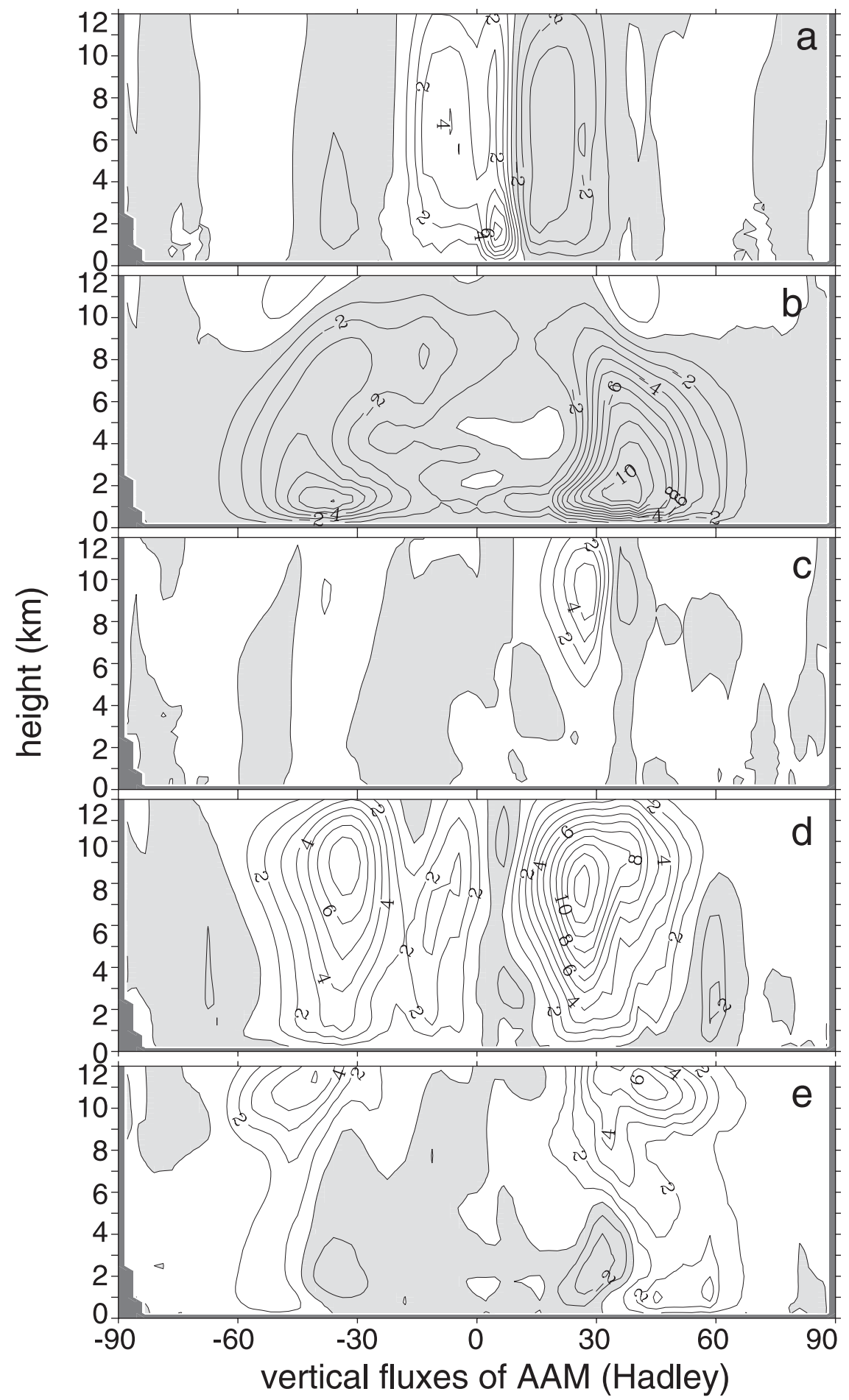

FIG. 2. Vertical fluxes of AAM with respect to zonal rings of 250-m depth in winter: (a) $F_{v 1} \sim \bar{w} \bar{\rho} \bar{m}$ in 20.0 Hadley, (b) $F_{v 2} \sim \overline{w^{\prime} \rho^{\prime}} \bar{m}$ in 0.1 Hadley, (c) $F_{v 3} \sim \bar{w} \overline{u^{\prime} \rho^{\prime}} a \cos \varphi$ in 0.002 Hadley, (d) $F_{v 4} \sim \overline{u^{\prime} w^{\prime}} \bar{\rho} a \cos \varphi$ in 0.1 Hadley, and (e) $F_{v 5} \sim \overline{u^{\prime} w^{\prime} \rho^{\prime}} a \cos \varphi$ in 0.001 Hadley.

and weakly positive near the Poles, $\psi$ at $z=0$ will increase from the equator northward with a maximum at $\sim 30^{\circ} \mathrm{N}$, and decrease with a minimum near $60^{\circ} \mathrm{N}$. As demonstrated by Oort and Peixoto (1983), it is not necessary to solve (3.3). One may integrate (3.1) upward given the profile of $\psi$ at the bottom. This method is correct 


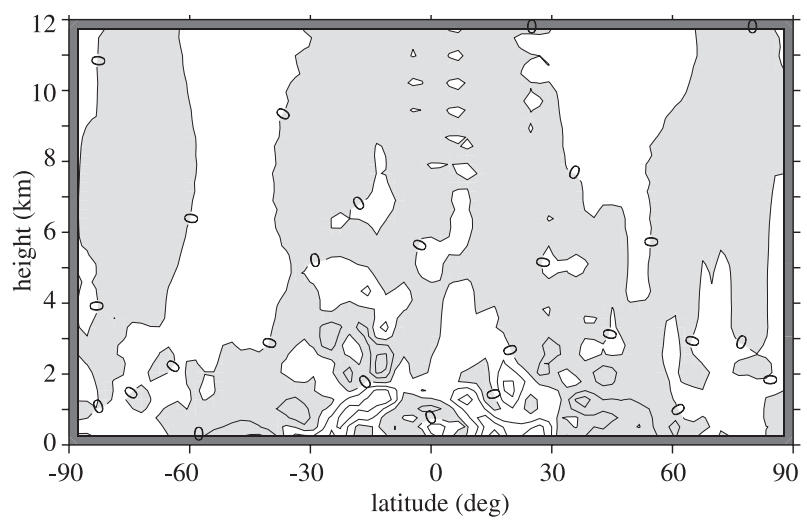

FIG. 3. Spurious flux differences in 0.5 Hadley as obtained by computing the left-hand side of the flux divergence in (1.10). The field is smoothed by applying a nine-point smoother.

and simple with perfect data. The more complicated approach pursued here has the advantage that equal weight is given to the horizontal and vertical flux data. Moreover, the observed profile of $\psi$ can be imposed on top. The actual calculations have been performed for a gridpoint function $\widetilde{\psi}_{i j}$, where the index $i(j)$ covers the meridional (vertical) direction. Moreover, (3.3) is replaced by the discrete formula:

$$
\begin{aligned}
& \widetilde{\psi}_{i+1 j}+\widetilde{\psi}_{i-1 j}+\widetilde{\psi}_{i j+1}+\widetilde{\psi}_{i j-1}-4 \widetilde{\psi}_{i j} \\
& =F_{v i j}-F_{v i-1 j}-F_{m i j}+F_{m i j-1} .
\end{aligned}
$$

This has the advantage that the observed total fluxes can be obtained by forming simply differences so that, for example,

$$
F_{m i j}=\widetilde{\psi}_{i j}-\widetilde{\psi}_{i j+1}
$$

Note that the arguments of the functions $F_{m}$ and $F_{v}$ in (1.6) and (1.7) have been replaced by the gridpoint indices, which implies $\varphi_{2}-\varphi_{1}=D \varphi, z_{2}-z_{1}=D z$.

The resulting streamfunction pattern in Fig. 4a is, of course, similar to that in Oort and Peixoto (1983) with a dominant anticyclonic Hadley cell and two adjacent cyclonic cells. There is one more anticyclonic cell in the Southern Hemisphere. It is clear from Figs. 1 and 2 that $F_{m 1}$ and $F_{v 1}$ are the main contributors to this pattern. Also given in Fig. $4 \mathrm{c}$ is the profile of the sum of both torques calculated for belts of width $D \varphi$. These "fluxes" through the surface are rather small when compared to those in the free atmosphere. Accuracy becomes a problem as can be seen immediately in the equatorial region. The position of the 50 Hadley contour (bold) near the equatorial surface in Fig. 4 is influenced by the vertical resolution. This affects the dense packing of the streamlines emanating from the surface. They have to ascend in a fairly narrow sheet centered at $\sim 15^{\circ} \mathrm{S}$ to split in the upper troposphere and lower stratosphere. One branch turns northward well above the Hadley cell and descends at $30^{\circ} \mathrm{N}$. The other branch turns southward and descends near $\sim 35^{\circ} \mathrm{S}$. The "sinks" in midlatitudes are reached within relatively shallow layers. These layers are better visible in Fig. 4b. They may be resolved reasonably well with a vertical grid distance of $250 \mathrm{~m}$, but we cannot be sure which processes are responsible for the final descent to the surface. Figure 2 suggests that this final descent is closely linked to the vertical transient mass flux $F_{v 2}$, but the motion of these mass fluxes parallel to the main isentropes speaks against this idea. Moreover, the omission of the turbulent stress $\tau$ in the free atmosphere is rather doubtful in the equatorial domain where convection transports AAM vertically (e.g., Rosenlof et al. 1986), so that the location of the streamlines is somewhat uncertain. Moreover, the kinks of the lines $\psi=0$ close to the ground near $40^{\circ} \mathrm{S}$ and $40^{\circ} \mathrm{N}$ are not due to observations of downward fluxes, but reflect the prescribed negative torques at the bottom. Finally, the shallow flux layer near the ground in midlatitudes is at least partly immersed in the planetary boundary layer where $\tau$ is dominant. We are, therefore, not able to give a conclusive description of the paths of the AAM flux from the tropical sources to the midlatitude sinks. Moreover, it is not obvious how the negative torques north of $45^{\circ} \mathrm{N}$ can be balanced by fluxes out of the tropics. In particular, there is no evidence that the convergence of eddy transports $F_{m 4}$ at midlatitudes is needed to balance the AAM losses at the surface in contrast to the standard concept mentioned in the introduction.

\section{Discussion and conclusions}

This investigation has been partly motivated by the surprising fact that no direct evaluation of the mean vertical eddy flux of AAM has been published as yet. The $z$ system has been chosen for this work because eddy fluxes of mass are dealt with explicitly in this system. Moreover, treatment of the lower boundary is not problematic in contrast to $\theta$ and $p$ systems at least over the oceans. This enables us to determine an AAM streamfunction although a reasonably accurate evaluation of fluxes is impossible over mountainous terrain. Moreover, there are losses of accuracy due to the interpolations from the hybrid ERA coordinate system. In particular, it is well known that mass continuity is sensitive to even small errors in the velocity components.

The switch to the $z$ system implies that five fluxes have to be computed, which had partly not been evaluated before. Out of these the triple terms are clearly unimportant. There is, however, a transient eddy density flux that appears to be approximately "adiabatic." This flux is 

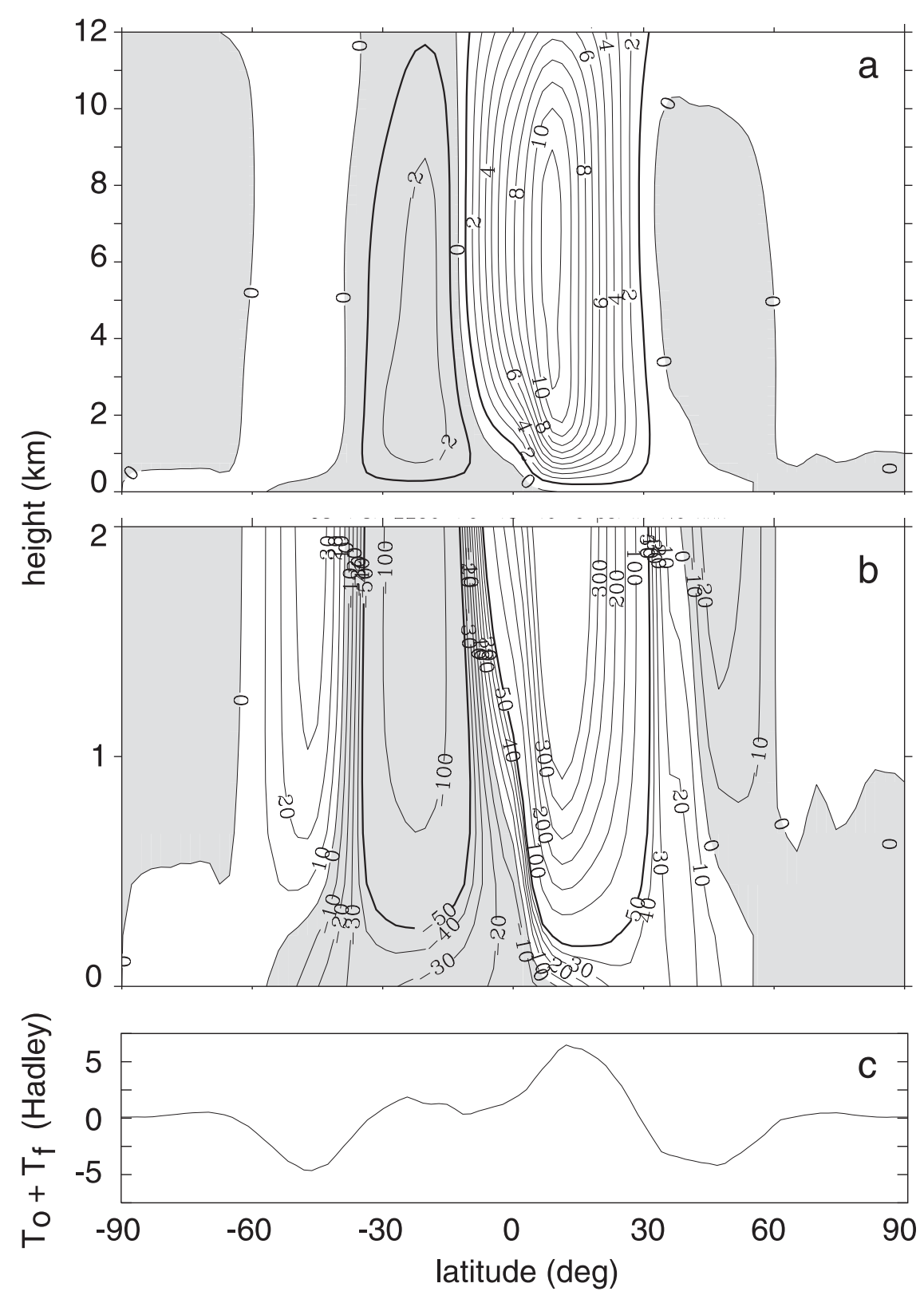

FIG. 4. Gridpoint streamfunction $\widetilde{\psi}$ in Hadley: (a) total domain with a contour interval of 50 Hadley, (b) lowest $2 \mathrm{~km}$ with a contour interval of 1 Hadley, and (c) total mean torque $\overline{T_{o}}+\overline{T_{f}}$ in Hadley. Bold lines in (a),(b) signify the 50 Hadley contour.

of the same order of magnitude as the better-known transient fluxes of relative AAM that are also approximately adiabatic. These mass fluxes bear witness of the baroclinic activity at the midlatitudes.

The streamfunction pattern comes close to that of Oort and Peixoto (1983) and suggests that the AAM gained in the tropical belt at the surface is transported upward in the Hadley cell and descends in both hemispheres mainly via the mean circulation cell. The final descent to the ground remains unclear because of the omission of the subgrid turbulent transports in the boundary layer. This omission is also problematic in the tropical convection zone where "cumulus friction" appears to be particularly important (e.g., Rosenlof et al. 1986). Thus, details are open, but there is no evidence that the losses in the midlatitudes by friction and mountains are balanced by the meridional eddy transports out of the tropics.

Acknowledgments. Comments by the anonymous reviewers helped to improve the paper. 


\section{REFERENCES}

Egger, J., 2006: The moisture torque. Meteor. Z., 15, 671-673.

$\longrightarrow$, and K.-P. Hoinka, 2010: Regional contributions to isentropic pressure torques. Mon. Wea. Rev., 138, 4175-4185.

Gallimore, R., and D. Johnson, 1981: The forcing of the meridional circulation of the isentropic zonally averaged circumpolar vortex. J. Atmos. Sci., 38, 583-599.

Hantel, M., and J. Hacker, 1978: On the vertical eddy transports in the Northern Atmosphere. 2. Vertical eddy momentum transport for summer and winter. J. Geophys. Res., 83, 1305-1318.

Held, I., and T. Schneider, 1999: The surface branch of the zonally averaged mass transport circulation in the troposphere. J. Atmos. Sci., 56, 1688-1697.

Holton, J., 1992: An Introduction to Dynamic Meteorology. Academic Press, 511 pp.

Johnson, D., 1980a: A generalized transport equation for use with meteorological coordinate systems. Mon. Wea. Rev., 108, 733-745.
_ 1980b: The forcing and maintenance of global monsoonal circulations: An isentropic analysis. Advances in Geophysics, Vol. 31, Academic Press, 43-329.

Oort, A., and J. Peixoto, 1983: Global angular momentum and energy balance requirements from observations. Advances in Geophysics, Vol. 25, Academic Press, 355-490.

Peixoto, J., and A. Oort, 1992: Physics of Climate. American Institute of Physics, $320 \mathrm{pp}$.

Rosenlof, K., D. Stevens, J. Anderson, and P. Cieselski, 1986: The Walker Circulation with observed zonal winds, a mean Hadley cell, and cumulus friction. J. Atmos. Sci., 43, 449467.

Starr, V., and R. White, 1951: A hemispherical study of the atmospheric angular-momentum balance. Quart. J. Roy. Meteor. Soc., 77, 215-225.

Trenberth, K., 1987: The role of eddies in maintaining the westerlies in the Southern Hemisphere winter. J. Atmos. Sci., 44, 1498-1508. 